\title{
Veterinary Telemedicine during the pandemic SARS-CoV-2
}

\author{
Abelardo Morales-Briceño $0^{1,2,5 *}$, Jimena Martínez ${ }^{2}$, José Fuentes-Acevedo ${ }^{3}$, \\ Carolina Ibarra ${ }^{3}$, Oscar Redondo ${ }^{4}$, Rossdalyn Rosales ${ }^{4}$, Kate Alteneiji ${ }^{5}$ and \\ Faiza Falaknaz ${ }^{5}$ \\ ${ }^{1}$ Alshula Veterinary Pharmacy for Veterinary Service, Doha, Qatar \\ ${ }^{2}$ VetCase, Montevideo-Uruguay \\ ${ }^{3}$ Veterinaria Patagonia, Puerto Natales, XII Región, Chile \\ ${ }^{4}$ Consultas Altos Mirandinos, Caracas-Venezuela \\ ${ }^{5}$ Kate Equine Physiotherapy Services. Fujairah, United Arab Emirates
}

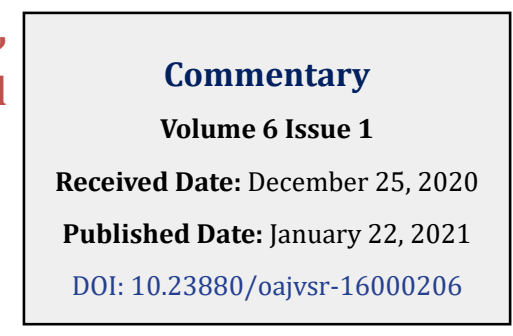

*Corresponding author: Abelardo Morales-Briceño, Alshula Veterinary Pharmacy for Veterinary Service, Doha, Qatar, Email: aamorales13@gmail.com

\section{Commentary}

Telehealth, spurred by the rise of digital communications technologies now, presents both opportunities and challenges for healthcare providers, including veterinarians and their staff. Telemedicine will in time become an integral part of the practice of certain aspects of veterinary medicine [1]. Understanding key definitions is important as you decide whether and how to implement telemedicine in your veterinary practice [2]. In veterinary practice, a Digital Veterinary Diagnostic teleconsultancy service has been established [1], we have some experiences in the last years in Chile, Kuwait, Qatar, United Arab Emirates, Uruguay and Venezuela, a market that grows every year. The current situation related to the SARS-CoV-2 associated pandemic represents a great opportunity to develop telehealth in veterinary medicine. Telemedicine is a tool of practice, not a separate discipline within the profession. The appropriate application of telemedicine can enhance animal care by facilitating communication, diagnostics, treatments, client education, scheduling, and other tasks [2]. With intensifying concern around COVID-19, use of telemedicine has become an important way to protect and monitor the health of veterinary patients and veterinary teams. Using telemedicine can help prevent the spread of COVID-19, because it allows veterinary patients to be appropriately triaged and monitored with only those veterinary patients that really need to be seen making the trip to the clinic along with their owners [2]. Telemedicine must be conducted within an existing veterinarian-clientpatient relationship (VCPR) - this is a key point to which the entire veterinary team, must follow. The only exception is surrounding an emergency - advice given in an emergency situation - until that patient is seen or transported to a veterinarian [3]. Telemedicine is a subcategory of telehealth that involves use of a tool to exchange veterinary information electronically from one site to another to improve a patient's clinical health status [2]. Telemedicine can be broadly defined the use of telecommunications technologies to provide medical information and services [4]. Veterinary Telemedicine is also seen as providing efficient and convenient access for busy working people, s system that started as a simple transfer of images has blossomed into a complex array of technologies that form electronic veterinary networks to provide remotely assisted surgery, diagnostic or therapeutic consultations, case management and education [2]. Veterinarian practitioners must comply with laws and regulations in the state in which they are licensed to practice veterinary medicine, in most countries telemedicine has not been regulated, however in the future it is legislation to be developed. The two subcategories of telehealth that are most developed in veterinary medicine are: teleconsulting and telemonitoring. Teleconsulting is a subcategory of telehealth in which a general practice veterinarian uses telehealth tools to communicate with a veterinary specialist to gain insights and advice on the care of a patient. In veterinary pathology has developed in recent years, the consultation of clinical-pathological cases with specialists from each area worldwide. The importance of Veterinary Telemedicine to break down geographical barriers and bring specialized care to places that if it were not through Telemedicine would be 


\section{Open Access Journal of Veterinary Science \& Research}

impossible, maintaining the quality of specialized veterinary care. One advertisement promises instant connection to a veterinarian $r$ from your phone, tablet, or computer at any time. The owner opens the app, describes her/his symptoms of the animal and requests to see a veterinarian. You are connected to a veterinarian, with whom you consult by text, audio, or video. The vet can make a diagnosis and, if necessary, provide prescriptions. Veterinary telemedicine is following along the same path as human telemedicine but has challenges associated with the inability of patients to describe their ailment. Telemonitoring is remote monitoring of patients who are not at the same location as the health care provider. This could range from the use of a portable glucose monitor to a wearable monitoring device that captures the patient's vital signs and other behaviors. A great development has been observed by practicing veterinarians in the last 5 years, coupled with the rise of social networks, since it allows continuous monitoring of the evolution of a patient. Recently in many farms and veterinary hospitals closed TV circuits are kept to continuously monitor animals in stables, boxes, even in hospitalization. There appears to be a bright future for telemedicine for animals. It expands access to veterinary health care, provides an inexpensive triage service, provides payment for veterinary consultation, and is particularly effective in situations such as follow-up after an office or hospital visit or for inspection of surgical sites or mobility [4]. In the last 10 months after the declared SARS-CoV-2 pandemic by the WHO, on March 12, 2020, telemedicine has been developed as an educational tool, most of the Universities and international professional associations have developed within their platforms Continuous education update tools for veterinarians and veterinary medicine students, through some platforms such as zoom, YouTube, Google, Microsoft, including WhatsApp, among others. This represents a breakthrough for veterinary medicine worldwide.

\section{References}

1. Marsa M, Auerb REJ (2006) Telemedicine in veterinary practice. Jl S Afr vet Ass 77(2): 75-78.

2. Veterinary telehealth: The basics. American Veterinary Medical Association.

3. Burns K (2020) COVID-19, Telemedicine and Veterinary Nurses.

4. Gyles C (2019) Veterinary telemedicine. Can Vet J 60(2): 119-122.

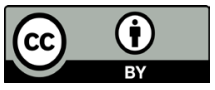

